\title{
LBHNC: A LUNAR-BASED HEAVY NUCLEUS DETECTOR
}

\author{
M.H. Salamon \\ Physics Department, University of Utah, Salt Lake City, UT 84112 \\ P.B. Price \\ Physics Department, University of California, Berkeley, CA 94720
}

G. Tarlé

Physics Department, University of Michigan, Ann Arbor, MI 48109

\section{ABSTRACT}

A passive, large-area experiment for the detection of cosmic ray actinides on the lunar surface is discussed. Due to the absence of a geomagnetic cutoff, a $100 \mathrm{~m}^{2}$ array of nucleartrack-detecting glass plates in 5 years will detect $-300-1000 \mathrm{U}$ and Th cosmic ray nuclei of energies $>0.85 \mathrm{GeV} / \mathrm{u}$ (compared to the present world's total of 4 actinides). With a charge resolution at uranium of $-0.25 e$, the $U / T h$ ratio can be accurately determined, thereby dating the $r$-process component of the cosmic rays; the presence of a fresh $r$-process component would be corroborated by the likely detection of transuranics as well. In addition, abundances in the $\mathrm{Pt} / \mathrm{Pb}$ and sub- $\mathrm{Pt} / \mathrm{Pb}$ regions and abundances of secondary actinides would provide detailed data on the $0.1 \mathrm{~g} / \mathrm{cm}^{2}$ region of the cosmic ray path length distribution, hence on the astrophysical site of origin of these cosmic rays. Finally, should a fresh $r$-process component exist, the detection of postulated superheavy nuclei is conceivable. With an analys is station at the Lunar Base, glass plates could periodically be harvested, analyzed, annealed/remelted, and replaced onto the lunar surface.

\section{INTRODUCTION}

Data from the Danish-French instrument ${ }^{1}$ and the Heavy Nuclei Experiment ${ }^{2}$ on board HEAO-3, and the Ariel 6 satellite $^{3}$ show that for $Z<60$ the galactic cosmic ray (CR) elemental distribution is very similar to that of the solar system (SS), after the effects of cosmic ray propagation have been taken into account. However, for $Z>60$, data from the Heavy Nuclei Experiment and Ariel-6 instruments suggest that there may be a significant enhancement of $r$-process elements in the ultra-heavy component of the $C R^{\prime} s^{2}$, raising the possibility that a fraction of this component may be freshly synthesized nuclei from recent supernovae, accelerated to $C R$ energies by the supernovae shocks.

A high-statistics measurement of the $C R$ actinide abundances would definitively answer this question. Figure 1 (taken from Ref. 5) shows the abundances of the actinide elements from Z-90 to 96 as a function of time after explosive nucleosynthesis. Since the propagation time of $\mathrm{CR}$ actinides 


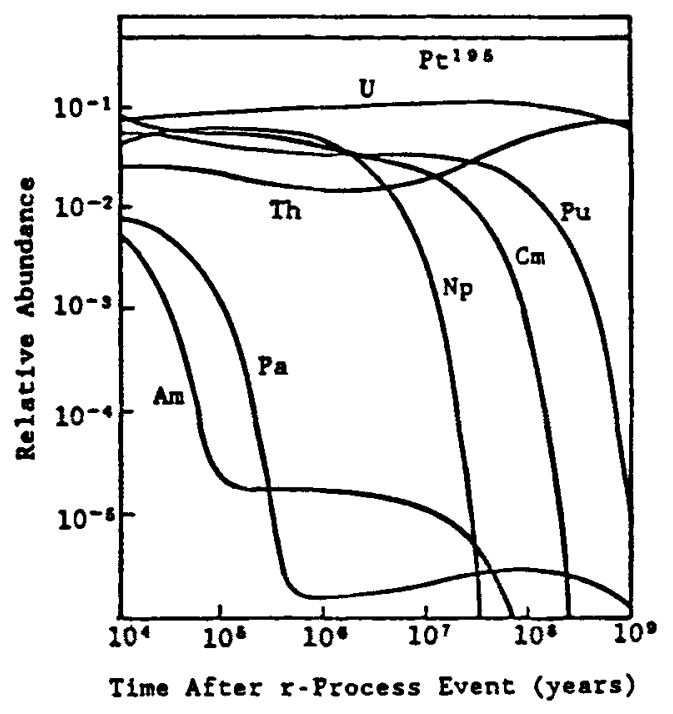

Fig. 1 should be less than $10^{7}$ yr (nuclear interactions reduce the lifetime from the CR lifetime of $-10^{7}$ yr derived from ${ }^{10} \mathrm{Be}$ data), the $C R U / T h$ ratio is an excellent indicator of CR age since nucleosynthesis; the measurement of several hundred $\mathrm{U}+\mathrm{Th} C \mathrm{CR}^{\prime} \mathrm{s}$ would give a $U / T h$ ratio to -108 accuracy, sufficient to determine the epoch of $\mathrm{CR}$ nucleosynthesis. In addition, the observation of short-lived transuranics such as $\mathrm{Cm}$ and $\mathrm{Pu}$ would be an unambiguous signature of a fresh $r$-process enhancement of the CR's,

and would provide strong evidence for the role of shocks in CR acceleration.

A detector that is also capable of cleanly separating even and odd charges in the ( $\mathrm{Pt}$ to $\mathrm{Pb}$ )/sub ( $\mathrm{Pt}$ to $\mathrm{Pb}$ ) region and beyond would dramatically improve our knowledge of the CR path length distribution (PLD) in the critical region of $0-1 \mathrm{~g} / \mathrm{cm}^{2}$, thereby yielding information on the astrophysical sites of origin of the CR's. To simultaneously fit CR secondary/primary ratios $B / C$ and sub-Fe/Fe, an energy-dependent deficit in short (0-1 $\mathrm{g} / \mathrm{cm}^{2}$ ) pathlengths in the CR PLD is required 6 , supporting the picture of nested leaky box sources, such as $O B$ associations in dense molecular clouds. The secondaries of $\mathrm{Pt}-\mathrm{Pb}$ and $\mathrm{U}-\mathrm{Th}$ are ideally suited to study this region of the PLD because their mean nuclear interaction length is of the order of $1 \mathrm{~g} / \mathrm{cm}^{2}$ in hydrogen. An extraordinary charge resolution is required for this purpose: $<0.3 \mathrm{e}$ at a $\mathrm{Z}$ of -90 .

Finally, a high-statistics measurement of the ultraheavy $\mathrm{CR}^{\prime}$ 's could concelvably result in the first detection of superheavy nuclei $(Z>100)$. Should an island of nuclear stability exist beyond the transuranics with lifetimes of $>10^{7}$ yr, then a fresh r-process CR component might carry with itself the only extant superheavy nuclei accessible to us.

At the present time, the total number of observed $C R$ actinides is no more than four, detected with satellite instruments ${ }^{2},{ }^{3}$ of charge resolution $>$ one charge unit at uranium. A lunar base, by comparison, would allow the deployment of an array capable of detecting several hundred actinides with a charge resolution of $-0.25 e$. 


\section{LBHNC}

The recent discovery ${ }^{7}$ of the extraordinary properties of certain phosphate glasses as nuclear track detectors has made possible the construction of an extremely simple and powerful detector of ultraheavy CR's of $2>60$. Schott's BP-18 phosphate glass has remarkable immunity to many of the problems inherent in a long-duration space experiment involving nuclear track detectors. These include fading of latent tracks over exposure times of months or years at even fairly low temperatures ${ }^{2}$, the variation of sensitivity with registration temperature ${ }^{10}$ and oxygen pressure ${ }^{11}$, and the growth of latent track reactivity with time 12. BP-1 glass suffers no fading of latent tracks up to a test limit of 5 months at $50^{\circ} \mathrm{C}$, ensuring negligible fading over several years at a lunar base site. At the design operating temperature of $-20^{\circ} \mathrm{C}$, the dependence of track etch rate on registration temperature is remarkably weak, with a fractional shift in etch rate of only $-1 \times 10-3 /{ }^{\circ} \mathrm{C}$. Finally, the response of BP-1 is independent of oxygen pressure, so pressure vessels with their unavoidable mass overburden are unnecessary; also, there is no measurable variation of latent track reactivity with time.

The charge resolution of BP-1 glass is unequalled in the high-z regime. Figure 2 shows data on the fragmentation of 1

$\mathrm{GeV} / \mathrm{u} \mathrm{Au}$ ions at LBL's Bevalac.

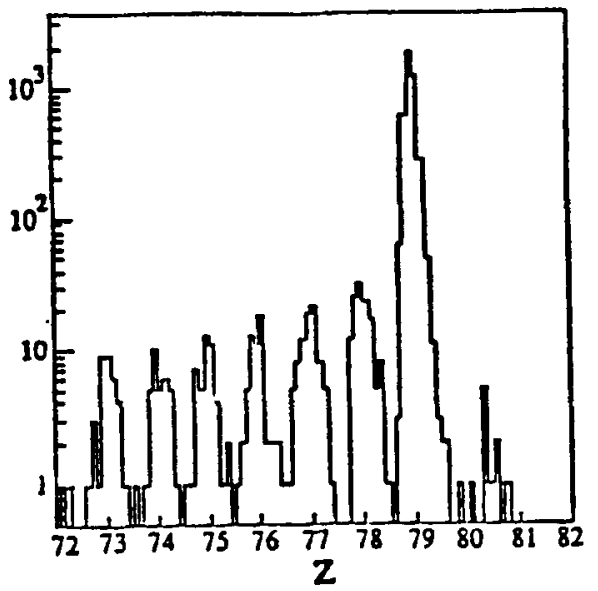

Fig. 2
A stack of 5 sheets of glass (10 surfaces) sufficed to yield a charge resolution of $0.06 \mathrm{e}$ (note that the ordinate in Fig. 2 is logarithmic)! With CR energies initially unknown, the charge resolution versus energy also depends upon the ability to uniquely fit response curves of individual elements to the measured track etch rate versus stack depth. Figure 3 shows the results of a Monte Carlo calculation for a stack of 14 sheets of $0.2 \mathrm{~cm}$-thick BP-1 glass. It is seen that a majority of events have their charge resolved to $<0.3 \mathrm{e}$.

A Lunar-Based Heavy Nucleus Detector (LBHNC) would consist of an array of BP-1 glass stacks totalling $100 \mathrm{~m}^{2}$ in area (and acceptance solid angle of $2.8 \mathrm{sr}$ ), deployed for an exposure period of 5 years, with each stack consisting of $160.2 \mathrm{~cm}$-thick sheets. A lunar site is arguably the ideal location for such an experiment. The absence of a lunar magnetic field implies the absence of any rigidity cutoff, resulting in substantially higher $C R$ fluxes on the Moon than in a low-inclination orbit about the 

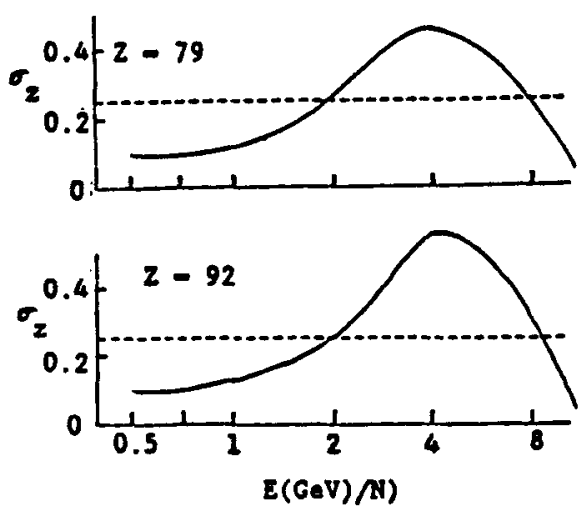

Fig. 3
Earth. The flux of $C R U$ and Th can be estimated from the measured $\mathrm{CR} F \mathrm{Fe}$ flux by scaling with the SS U+Th/Fe ratio, and accounting for propagation effects. The CR Fe flux measured by the Danish-French HEAO- 3 experiment ${ }^{1}$ has been corrected for the effects of its (variable) geomagnetic cutoff, resulting in a spectrum corresponding to that at 1 AU outside the Earth's magnetosphere ${ }^{13}$. This is then scaled by the sS abundance

ratio $4+\mathrm{U}+\mathrm{Th} / \mathrm{Fe}=5.1 \times 10-8$. Propagation effects are taken into account by calculating the survival fraction of both Fe primaries and $U+T h$ primaries after propagation through a truncated exponential PLD using the energy-dependent parameters of GarciaMunoz et al. ${ }^{6}$, and correcting for first-ionization-potential selection effects. Imposing an energy cut of $>0.85 \mathrm{GeV} / \mathrm{u}$ to ensure adequate range within a glass stack, we estimate that a total of $-350 \mathrm{U}+\mathrm{Th}$ nuclei (this does not include actinide secondaries) will be detected by LBHNC. Should the $r$-process enhancement suggested by the HEAO-C and Ariel- 6 data be real, this number might be as high as -1000 . In either case, these statistics would be sufficient to determine the $U / T h$ ratio to within $-10 \%$, and they imply that LBHNC would be sensitive to transuranics even if the fresh $r$-process component were to comprise only a few percent of the total ultraheavy $C R$ flux.

\section{ACKNOWLEDGEMENTS}

We wish to thank Dick Mewaldt for helpful conversations.

\section{REFERENCES}

[1] P. Goret et al., Proc. 18th ICRC (Bangalore) 9, 139 (1983), and references therein.

[2] W.R. Binns et al., Ap. J. 346, 997 (1989).

[3] P.H. Fowler et al., Ap. J. 314, 739 (1987).

[4] J.P. Meyer, Ap. J. Supp1. 57, 173 (1985).

[5] J.B. Blake and D.N. Schramm, Astrophys, and Sp. Sci. 30, 275 (1974).

[6] M. Garcia-Munoz et al., Ap. J. 280, L13 (1984).

[7] P.B. Price et al., Nuc1. Instr. Meth. B21, 60 (1987).

[8] Shicheng Wang et al., Nucl. Instr. Meth. (in press, 1989).

[9] M.H. Salamon et al., Nucl. Instr. Meth. B17, 173 (1986).

[10] A. Thompson et al., Proc. 11th Inter. Conf. on Solid State Nuclear Track Detectors (Bristol, 1981).

[11] J. Drach et al., Nucl. Instr. Meth. B23, 367 (1987). 
626 Lunar-Based Heavy Nucleus Detector

[12] J. Drach and P.B. Price, Nucl. Instr. Meth, B28, 275 (1987).

[13] E. Juliusson et al., Proc. 18th ICRC (Bangalore) 2, 21 (1985).

[14] A.G.W. Cameron, in Essays in Nuclear Astrophysics, ed. C.A. Barnes et al., Cambridge University Press (1982).

[15] M.A.I. Van Hollebeke et al., Proc. 19th ICRC (La Jolla) 4 , 209 (1985). 